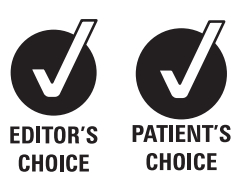

${ }^{1}$ Department of Psychiatry, Chinese University of Hong Kong, Hong Kong SAR, China; ${ }^{2}$ Department of Medicine and Therapeutics, Chinese University of Hong Kong, Hong Kong SAR, China; ${ }^{3}$ Department of Diagnostic Radiology and Organ Imaging, Chinese University of Hong Kong, Hong Kong SAR, China

Correspondence to:

Dr W K Tang, Department of Psychiatry, Shatin Hospital,

Shatin, NT, Hong Kong SAR,

China; tangwk@cuhk.edu.hk

Received 17 February 2009

Revised 24 May 2009

Accepted 27 May 2009

Published Online First

18 June 2009

\title{
Microbleeds and post-stroke emotional lability
}

\author{
W K Tang, ${ }^{1}$ Y K Chen, ${ }^{1,2}$ J Y Lu, ${ }^{1}$ V C T Mok, ${ }^{2}$ Y T Xiang, ${ }^{1}$ G S Ungvari, ${ }^{1}$ A T Ahuja, ${ }^{3}$ \\ K S Wong ${ }^{2}$
}

ABSTRACT

Objective: The clinical significance of microbleeds (MBs) in the development of psychiatric conditions following a stroke is unknown. Lesions located in various cortical and subcortical areas are thought to be involved in the pathophysiology of post-stroke emotional lability (PSEL). This study examined the association between PSEL and MBs.

Methods: A total of 519 Chinese patients with acute ischaemic stroke consecutively admitted to the acute stroke unit of a university affiliated regional hospital in Hong Kong were screened for PSEL 3 months after their index stroke. The number and location of MBs were evaluated with MRI.

Results: According to Kim's criteria, 74 (14.3\%) patients had PSEL. In comparison with the non-PSEL group, patients in the PSEL group were more likely to have MBs in the thalamus as a whole (16.2\% vs $6.5 \% ; p=0.004)$, its anterior $(6.9 \%$ vs $2.0 \%, p=0.02)$ and paramedian territories ( $8.1 \%$ vs $3.1 \% ; p=0.04)$, and a higher number of $\mathrm{MBs}$ in the entire brain $(1.7+3.4$ vs $1.3+5.0$; $p=0.031)$. MBs in the thalamus remained an independent predictor of PSEL in the multivariate analysis, with an odds ratio of 4.7 ( $p=0.002$ ).

Conclusion: Our results suggest that MBs in the thalamus may play a role in the development of PSEL. The importance of MBs in PSEL and other psychiatric conditions in stroke survivors warrants further investigation.

Cerebral microbleeds (MBs) are focal deposits of haemosiderin that indicate prior micro-haemorrhages. $\mathrm{MBs}$ are related to cerebral amyloid angiopathy, hypertension and atherosclerosis. ${ }^{1}$

$\mathrm{MBs}$ are common in ischaemic stroke. ${ }^{2}$ They may provide useful diagnostic and prognostic information, with potential therapeutic implications for the treatment of stroke. ${ }^{3} \mathrm{MBs}$ are associated with advanced small artery disease of the brain ${ }^{4}$ and leukoaraiosis, ${ }^{5}$ and they may also predict recurrent stroke. ${ }^{6}$

$\mathrm{MBs}$ have generally been considered to be clinically silent. Recent evidence suggests that $\mathrm{MBs}$ may be one of the important factors that cause cognitive impairments in subcortical vascular dementia ${ }^{7}$ where they are associated with cognitive dysfunction, particularly executive dysfunction. ${ }^{8}$ The clinical significance of $\mathrm{MBs}$ in psychiatric conditions following a stroke remains unknown.

Emotional lability (EL) is defined as virtually uncontrollable episodes of laughter, crying or both. ${ }^{9}$ Laughing or crying occurs without a clear relationship to a stimulus or the outbursts can be triggered by minor, non-specific stimuli. In addition, patients may not experience any concomitant or subsequent change in feeling. EL is a distressing and embarrassing complaint for the patient, is often socially disabling ${ }^{10}$ and may interfere with rehabilitation. Other terms that have been used for this clinical syndrome include emotionalism, pathological laughing and crying, pseudobulbar affect, pathological emotionality and emotional incontinence..$^{1112}$ Common methods of evaluating EL are clinical interviews, chart reviews, the Pathological Laughing and Crying Scale ${ }^{13}$ and other questionnaires. ${ }^{11}$

EL affects $11-52 \%$ of all stroke survivors. ${ }^{11} 14$ Post-stroke emotional lability (PSEL) usually starts within weeks of a stroke, ${ }^{15}$ may last from 1 week to a few years, ${ }^{1516}$ has a fluctuating clinical course ${ }^{12}$ and may respond to selective serotonin reuptake inhibitors. ${ }^{17}$

The neuropathological substrate of EL has yet to be determined. Wilson ${ }^{18}$ proposed that it was caused by bilateral corticobulbar motor tract lesions. Recently, Parvizi and colleagues ${ }^{19}$ hypothesised that disconnection in the cortico-pontinecerebellar pathways may also be an important link in the pathogenesis. Black ${ }^{20}$ suggested three levels of injury (cortical, bulbar and hypothalamic) as possible causes of pathological laughter, with the common factor being disinhibition in the upper brainstem level. House and colleagues ${ }^{21}$ proposed that frontal lesions may produce disinhibition of emotional control similar to the way that frontal lobe damage produces disinhibition of social behaviour. EL has been found to co-occur with frontal release signs such as grasp reflex. ${ }^{22}$ Neuroimaging studies have shown that EL may be related to specific frontal areas, such as the anterior cingulate and dorsolateral prefrontal cortices $^{23}$ and the medial inferior frontal areas. ${ }^{24}$ PSEL is associated with anterior cortical, ${ }^{9}{ }^{25}$ frontal lobe ${ }^{26}$ and lenticulocapsular lesions, ${ }^{9}$ particularly those that involve the dorsal globus pallidus. ${ }^{27}$ Brainstem and parietal lesions can also generate EL. ${ }^{9} 2428$

The fronto-subcortical circuits are known to mediate emotion and affective behaviour. ${ }^{29}$ These circuits link specific areas of the frontal cortex to the striatum, basal ganglia and thalamus. ${ }^{30}$ Several lines of evidence suggest that the fronto-subcortical circuits are involved in the development of EL. ${ }^{23} 242627$ Thalamic lesions can give rise to a variety of neuropsychiatric complications following stroke, including disinhibition, affective changes, apathy, amnesia and dementia. ${ }^{31}$

The role of MBs in PSEL is unknown. The aim of this case control study was to assess the relationship between MBs and PSEL in stroke survivors.

\section{MATERIAL AND METHODS Subjects}

In total, 2337 patients with first ever or recurrent acute ischaemic stroke were admitted to the Acute 
Table 1 Demographic, clinical and MRI variables of subjects with and without post-stroke emotional lability

\begin{tabular}{|c|c|c|c|}
\hline Variable & PSEL $(n=74)$ & $\begin{array}{l}\text { No PSEL } \\
(\mathrm{n}=445)\end{array}$ & p Value \\
\hline Age (years)* & $65.6(9.9)$ & $65.6(12.0)$ & 0.9829 \\
\hline Female sex (n (\%)) & $38(51.4)$ & $168(37.8)$ & 0.027 \\
\hline Education (years) ${ }^{*}$ & $4.2(4.5)$ & $5.8(4.8)$ & 0.0089 \\
\hline MMSE score* & $24.8(3.6)$ & $26.3(3.1)$ & $<0.001 \dagger$ \\
\hline NIHSS score* & $5.4(3.8)$ & $4.3(3.2)$ & $0.004 \dagger$ \\
\hline Previous stroke (n (\%)) & $18(24.3)$ & $82(18.2)$ & $0.234 \%$ \\
\hline Presence of microbleeds (n (\%)) & $24(32.4)$ & $100(22.5)$ & 0.063 ; \\
\hline No of microbleeds & $1.7(3.4)$ & $1.3(5.0)$ & $0.031 \dagger$ \\
\hline \multicolumn{4}{|c|}{ Localisation of microbleeds in (n (\%)) } \\
\hline Frontal lobe & $9(12.2)$ & $27(6.1)$ & 0.056 \\
\hline Temporal lobe & $8(10.8)$ & $30(6.7)$ & $0.213 \%$ \\
\hline Parietal lobe & $8(10.8)$ & $33(7.4)$ & 0.316 \\
\hline Occipital lobe & $2(2.7)$ & $16(3.6)$ & $1.000 \S$ \\
\hline Basal ganglia & $8(10.8)$ & $39(8.8)$ & $0.570 \%$ \\
\hline Thalamus as whole & $12(16.2)$ & $29(6.5)$ & $0.004 \%$ \\
\hline Anterior territory & $5(6.9)$ & $9(2.0)$ & $0.020 \%$ \\
\hline Paramedian territory & $6(8.1)$ & $14(3.1)$ & $0.040 \%$ \\
\hline Lateral territory & $6(8.1)$ & $17(3.8)$ & 0.097 \\
\hline Posterior territory & $4(5.4)$ & $10(4.2)$ & $0.125 \S$ \\
\hline Brainstem & $8(10.8)$ & $26(5.8)$ & $0.110 \%$ \\
\hline Cerebellum & $5(6.8)$ & $31(7.0)$ & $1.000 \S$ \\
\hline
\end{tabular}

*Values are mean (SD).

†Mann-Whitney U test; $₫ \chi^{2}$ test, §Fisher exact test; $\bullet$ t test

MMSE, Mini-Mental State Examination; NIHSS, National Institute of Health Stroke Scale; PSEL, post-stroke emotional lability.

Stroke Unit of the Prince of Wales Hospital from December 2004 to June 2007. The Prince of Wales Hospital is a university affiliated general hospital serving a population of 800000 in Hong Kong. Of these, 874 of the admitted stroke patients received an MRI examination. Of patients with an MRI, 519 (59.4\%) were recruited from patients admitted with a first ever or recurrent ischaemic stroke. The inclusion criteria for the study were: (1) Chinese ethnicity; (2) age 18 years or above; (3) well documented (clinical presentation and CT scan of the brain) first or recurrent acute stroke occurring within 7 days before admission; (4) Cantonese as the primary language; and (5) ability and willingness to give consent. Exclusion criteria included: (1) transient ischaemic attacks, since $\mathrm{MBs}$ are rare in transient ischaemic attacks ${ }^{4}$; (2) cerebral haemorrhage, subdural haematoma or subarachnoid haemorrhage; and (3) history of a central nervous system disease such as tumour, trauma, hydrocephalus, multiple sclerosis, Parkinson's disease, etc. All participants were screened for PSEL by a qualified psychiatrist (WKT).

The study protocol was approved by the Clinical Research Ethics Committee of the Chinese University of Hong Kong. Consent of all subjects was obtained in accordance with the Declaration of Helsinki.

\section{Collection of demographic and clinical data}

A research nurse, who was blind to the psychiatrist's diagnoses, collected the demographic data (age, sex and education level) and assessed the severity of stroke with the National Institute of Health Stroke Scale (NIHSS). ${ }^{32}$

\section{Assessment of PSEL}

Psychiatric interviews were conducted 3 months after the stroke at a research clinic. A psychiatrist (WKT) administered a brief questionnaire to all participants. The diagnostic criteria of PSEL were based on those proposed by Kim. ${ }^{27}$ PSEL was considered to be present if patients exhibited excessive or inappropriate laughing, crying or both compared with their premorbid state. If the patient agreed that excessive or inappropriate laughing or crying occurred on two or more occasions since the latest episode of stroke, the diagnosis of PSEL was established. A research assistant (RA), trained by the principal author, administered the Chinese version of the MiniMental State Examination $(\mathrm{MMSE})^{34}$ to measure the participants' global cognitive function.

\section{Radiological examination}

MRI, including diffusion weighted imaging (DWI) and sequence of proton density, was performed on each participant with a 1.5 T system (Sonata, Siemens Medical, Erlangen, Germany) within 7 days of admission.

DWI spin echo EPI (TR/TE/excitation $=180 / 122 / 4$, matrix $=128 \times 128, \quad F O V=230 \mathrm{~mm}$, slice thickness/ gap $=5 \mathrm{~mm} / 1 \mathrm{~mm}$, EPI factor $=90$, acquisition time $=55 \mathrm{~s}$ ) with three orthogonally applied gradients were used with a b value of 1000 and 500. Axial gradient echo images were acquired as the second sequence with imaging parameters of TR/TE/ excitation $=350 / 30 / 2$, flip angle of $30^{\circ}$, slice thickness/ gap $=5 \mathrm{~mm} / 0.5 \mathrm{~mm}, \mathrm{FOV}=230 \mathrm{~mm}$, matrix $256 \times 256$ and acquisition time $=5 \mathrm{~min} 4 \mathrm{~s}$. Axial SE T1 (TR/TE/excitation $=425 / 14 / 2$, FOV $=230 \mathrm{~mm}$, slice thickness $/ g a p=5 \mathrm{~mm} /$ $0.5 \mathrm{~mm}$, matrix $=256 \times 256$ and acquisition time $=4 \mathrm{~min} 28 \mathrm{~s}$ ) and TSE T2 (TR/TE/excitation $=2500 / 120 / 1$, turbo factor of $15, \quad F O V=230 \mathrm{~mm}$, slice thickness $/$ gap $=5 \mathrm{~mm} / 0.5 \mathrm{~mm}$, matrix of $256 \times 256$ and acquisition time $=1 \mathrm{~min} 39 \mathrm{~s}$ ) images were also acquired.

A neurologist (YKC), who was blind to the psychiatric diagnoses, assessed the MRIs, which included the following.

1. Brain infarcts. Acute infarcts affecting the frontal, temporal, parietal and occipital lobes, coronary radiata, centrums semiovale, internal capsule, basal ganglia, thalamus, 
Table 2 Demographic, clinical and magnetic resonance imaging variables of subjects with and without poststroke emotional lability

\begin{tabular}{|c|c|c|c|}
\hline Variable & $\begin{array}{l}\text { PSEL } \\
(n=74)\end{array}$ & $\begin{array}{l}\text { No PSEL } \\
\text { ( } \mathrm{n}=445)\end{array}$ & p Value \\
\hline No of acute infarcts* & $1.5(2.2)$ & $1.4(2.1)$ & $0.604 \uparrow$ \\
\hline Volume of acute infarct $\left(\mathrm{cm}^{3}\right)^{*}$ & $4.0(16.1)$ & $1.6(4.3)$ & $0.200 \dagger$ \\
\hline \multicolumn{4}{|l|}{ Localisation of acute infarcts in ( $\mathrm{n}(\%))$} \\
\hline Frontal lobe & $7(9.5)$ & $25(5.6)$ & $0.203 \$$ \\
\hline Temporal lobe & $2(2.7)$ & $16(3.6)$ & $0.698 \S$ \\
\hline Parietal lobe & $4(5.4)$ & $30(6.7)$ & $0.804 \S$ \\
\hline Occipital lobe & $3(4.1)$ & $9(4.0)$ & $0.392 \S$ \\
\hline Basal ganglia & $18(24.3)$ & $90(20.2)$ & $0.421 \%$ \\
\hline Thalamus & $3(4.1)$ & $26(5.8)$ & $0.784 \S$ \\
\hline Brain stem & $14(18.7)$ & $61(13.7)$ & $0.238 \%$ \\
\hline Cerebellum & $1(1.4)$ & $7(1.6)$ & $1.000 \S$ \\
\hline Coronary radiate & $9(12.2)$ & $62(13.9)$ & $0.682 \ddagger$ \\
\hline Centrums semiovale & $4(5.4)$ & $19(4.3)$ & $0.555 \S$ \\
\hline Internal capsule & $11(14.9)$ & $59(13.3)$ & $0.708 \%$ \\
\hline No of old infarcts* & $0.9(1.5)$ & $0.9(1.7)$ & $0.977 \dagger$ \\
\hline \multicolumn{4}{|l|}{ Location of old infarcts (n (\%)) } \\
\hline Frontal lobe & $1(1.4)$ & $10(2.2)$ & $1.000 \S$ \\
\hline Temporal lobe & $2(2.7)$ & $8(1.8)$ & $0.641 \S$ \\
\hline Parietal lobe & $3(4.1)$ & $8(1.8)$ & $0.197 \S$ \\
\hline Occipital lobe & $1(1.4)$ & $7(1.6)$ & $1.000 \S$ \\
\hline Basal ganglia & $18(24.3)$ & $90(20.2)$ & $0.421 \%$ \\
\hline Thalamus & $7(9.5)$ & $27(6.1)$ & $0.275 \%$ \\
\hline Brain stem & $2(2.7)$ & $19(4.3)$ & $0.753 \S$ \\
\hline Cerebellum & $0(0.0)$ & $6(1.3)$ & $0.601 \S$ \\
\hline Coronary radiate & $9(12.2)$ & $42(9.4)$ & $0.466 \$$ \\
\hline Centrums semiovale & $3(4.1)$ & $11(2.5)$ & $0.434 \S$ \\
\hline Internal capsule & $4(5.4)$ & $25(5.6)$ & $1.000 \S$ \\
\hline Lacunar infarcts & & & $0.573 \%$ \\
\hline Presence of lacunar infarcts ( $\mathrm{n}(\%))$ & $44(59.5)$ & $249(56.0)$ & $0.626 \dagger$ \\
\hline No of lacunar infarcts* & $1.4(1.8)$ & $1.3(1.9)$ & \\
\hline \multicolumn{4}{|l|}{ White matter lesions } \\
\hline DWMH score* & $1.09(0.90)$ & $1.01(0.80)$ & $0.469 \dagger$ \\
\hline PVH score* & $1.23(0.71)$ & $1.24(0.84)$ & $0.962 \uparrow$ \\
\hline
\end{tabular}

*Values are mean (SD).

$\dagger$ Mann-Whitney U test; $\$ \chi^{2}$ test; $\S$ Fisher exact test.

DWMH, deep white matter hyperintensities; MMSE, Mini-Mental State Examination; NIHSS, National Institute of Health Stroke Scale; PSEL, post-stroke emotional lability; PVH, periventricular hyperintensities.

brainstem and cerebellum were recorded. ${ }^{35}$ The presence or absence of acute infarcts in these locations was recorded. Multiple infarcts or infarct(s) involving more than one location were counted in all locations they occurred. The total area of acute infarcts on DWI was measured with manual outlines, with restricted water diffusion identified on diffusion weighted images with $b$ values of 1000. The total volume was calculated by multiplying the total area by the sum of the slice thickness and gap.

2. MBs. MBs were defined as small $(2-10 \mathrm{~mm})$ hypointense lesions on T2 weighted gradient echo sequence but symmetric basal ganglia calcification and flow void artefacts of the pial blood vessels were excluded. ${ }^{36}$ The locations of MBs included the frontal, parietal, temporal and occipital lobes, basal ganglia, thalamus as a whole and its anterior, paramedian, lateral and posterior territories, ${ }^{37}$ brainstem and cerebellum. The number of MBs in each location was recorded. Inter-rater and intra-rater reliability tests of the measurement of MBs were performed on 30 participants. Inter-rater and intra-rater agreement was good (presence of MBs: inter-rater kappa $=0.78$; intra-rater $\mathrm{kappa}=0.85$; number of MBs: inter-rater intraclass correlation coefficient $($ ICC $)=0.91$; intra-rater ICC $=0.95$ )
3. White matter lesions (WML). The severity of WMLs was graded using the four point scale developed by Fazekas and colleagues. ${ }^{38}$ Periventricular and deep white matter hyperintensities were scored on axial proton density images. Inter-rater and intra-rater reliability tests were performed on 20 participants resulting in good agreement $($ kappa $=0.70-1.00)$.

\section{Statistical analysis}

All statistical tests were performed by SPSS for Windows (release 14.0; SPSS Inc, Chicago, Illinois, USA). Demographic and clinical variables (age, sex, education level, and NIHSS and MMSE scores) and the scores of the psychometric tests of the PSEL subjects were compared with those of matched controls using the $\chi^{2}$ test, Fisher's exact test, Student's $t$ test and the Mann-Whitney U test, as appropriate. Risk factors with a value of $p<0.10$ were then analysed by multivariate logistic regression analysis using a forward stepwise selection strategy. If the correlations between any of these putative risk factors were $\geqslant 0.50$, then additional models were examined to rule out colinearity. In the analysis, the odds ratio of any independent risk factor was interpreted as the risk of subsequent PSEL when all 


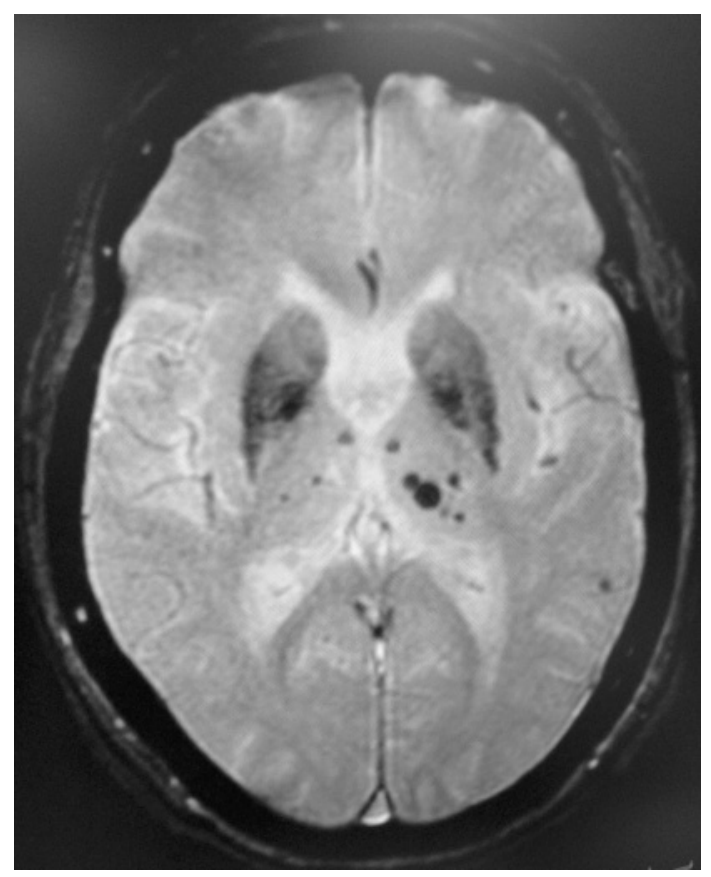

Figure $1 \mathrm{~T}^{*}$ weighted gradient echo MRI of a 78-year-old woman with post-stroke emotional lability shows multiple microbleeds in bilateral thalami (most in the left), ranging from 2 to $8 \mathrm{~mm}$ in diameter.

other risk factors were held constant. The level of significance was set at 0.05 .

\section{RESULTS}

Patient who were excluded from the study were older (73.9 (12.0) vs 66.0 (11.7) years; $p<0.001)$, were more likely to be female $(52.8 \%$ vs $39.3 \%$; $<0.001)$ and had a higher NIHSS score (10.1 (9.3) vs 4.5 (3.4); $\mathrm{p}<0.001)$.

Of the 519 patients screened, 74 (14.3\%) had PSEL. The demographic and MRI characteristics and stroke related data stratified by PSEL status are shown in tables 1 and 2 . The PSEL group had significantly more female subjects, a lower level of education and lower MMSE but higher NIHSS scores. The number of $\mathrm{MBs}$ was significantly higher in the PSEL group. MBs located in the thalamus were associated with PSEL, particularly the anterior and paramedian territories (fig 1). The number of $\mathrm{MBs}$ in the thalamus of subjects with PSEL ranged from 1 to 10 , with a mean of 3.2 (2.7). The association between MBs in the entire brain and in the frontal lobe and PSEL was of borderline significance. PSEL was not associated with the volume and location of infarcts, number of lacunar infarcts or the extent of WMLs.

Table 3 Multivariate logistic model of the clinical and radiological determinants of post-stroke emotional lability

\begin{tabular}{lrll}
\hline & \multicolumn{1}{l}{$\boldsymbol{\beta}$} & $\mathbf{p}$ Value & OR (95\% Cl) \\
\hline Microbleeds in thalamus & 1.542 & 0.002 & $4.676(1.743-12.544)$ \\
MMSE & -0.111 & 0.009 & $0.895(0.823-0.972)$ \\
NIHSS & 0.079 & 0.050 & - \\
Microbleeds in frontal lobe & 0.956 & 0.063 & - \\
Sex (female) & 0.517 & 0.076 & - \\
No of microbleeds & -0.090 & 0.093 & -
\end{tabular}

MMSE, Mini-Mental State Examination; NIHSS, National Institute of Health Stroke Scale.
MMSE score and education were correlated $(r=0.569$ $\mathrm{p}<0.01)$. The correlation between $\mathrm{MBs}$ in the thalamus and $\mathrm{MBs}$ in the entire brain was $0.524(\mathrm{p}<0.01)$. The following variables were entered into the regression model: sex, MMSE, NIHSS and $\mathrm{MBs}$ in the thalamus, and the frontal lobe and number of MBs. MBs in the thalamus and MMSE were significant independent predictors of PSEL. The odds ratio of $\mathrm{MBs}$ in the thalamus was 4.7 (table 3 ).

\section{DISCUSSION}

To the best of our knowledge, this was the first study to examine the role of $\mathrm{MBs}$ in stroke patients with EL. This observation challenges the prevailing view that microbleeds are clinically silent. ${ }^{4}$ The histopathological data revealed that $\mathrm{MBs}$ involve not only haemosiderin deposition but also affect the surrounding gliosis and cause frank necrosis or infarction, which means that they may be of clinical importance. ${ }^{39}$

The findings of this study suggest that microhaemorrhages are important components of the pathomechanism of PSEL. PSEL was associated with MBs in the thalamus, particularly the anterior and paramedian territories. The thalamus can be divided into different regions, according to the pattern of blood supply. The paramedian territory is supplied by the thalamoperforating arteries, which originate from the first part of the posterior cerebral artery. ${ }^{37}$ The paramedian thalamic territory has connections between cortical (prefrontal, orbitofrontal and mediofrontal cortex) and subcortical (ventral pallidum and internal globus pallidus) structures. ${ }^{40} 41$ Paramedian thalamic lesions can cause personality changes and disinhibition, ${ }^{42}$ such as crying spells, labile affect and even frank manic symptoms. ${ }^{43}$ It is thought that these behavioural symptoms were due to thalamofrontal disconnection. ${ }^{31} \mathrm{~A}$ wide range of behavioural and personality changes have been associated with lesions in the anterior territory, including euphoria, lack of emotional concern and emotional central facial paralysis. The latter is characterised by a facial weakness during emotional displays such as laughing or crying. ${ }^{44}$ Patients with executive dysfunction have more microbleeds in the frontal region. ${ }^{8}$ There is evidence suggesting that executive functions are impaired in patients with EL. ${ }^{44} 45$ Further exploration of the relationship between MBs in the frontal lobe, executive functions and PSEL is warranted.

The main limitation of this study is its relatively small sample size, which resulted in a low power in detecting the potential differences between groups with respect to frontal lobe MBs. Furthermore, the assessment of PSEL was made only once, at the 3 month follow-up. As patients who died before the 3 month follow-up were not included in the study, the sample was biased to an unknown degree. In addition, patients who could not give consent due to dementia or aphasia associated left side infarcts were excluded. The excluded group had more female patients, they were older and had more severe stroke. As cognitive impairment, ${ }^{21}$ left side lesions, ${ }^{21}$ female sex, ${ }^{9}$ younger age $^{14}$ and neurological deficits ${ }^{9}$ were associated with PSEL, this selection bias may limit the generalisability of the findings.

It is interesting to note that thalamic MBs, a pre-stroke lesion, can contribute to the development of an event such as EL after stroke. It is well known that pre-stroke abnormalities do contribute to post-stroke psychiatric problems, including dementia and depression. WMLs and cerebral atrophy predict post-stroke dementia. ${ }^{46}$ Similarly, WMLs predict post-stroke depressive symptoms. ${ }^{47}$

In conclusion, the results of this study suggest that $M B$ s at the thalamus may contribute to the development of PSEL. This assumption, while biologically plausible, should be regarded as 
speculative at this point. Further exploration of the importance of MBs in PSEL and other psychiatric sequels in stroke survivors is clearly warranted.

Funding: This work was supported by a direct grant for research 2005/2006 from the Chinese University of Hong Kong.

Competing interests: None.

Ethics approval: The study protocol was approved by the Clinical Research Ethics Committee of the Chinese University of Hong Kong.

Provenance and peer review: Not commissioned; externally peer reviewed.

\section{REFERENCES}

1. Vernooij MW, van der Lugt A, Ikram MA, et al. Prevalence and risk factors of cerebral microbleeds: the Rotterdam Scan Study. Neurology 2008;70:1208-14.

2. Werring DJ, Coward LJ, Losseff NA, et al. Cerebral microbleeds are common in ischemic stroke but rare in TIA. Neurology 2005:65:1914-18.

3. Cordonnier C, Al-Shahi Salman R, Wardlaw J. Spontaneous brain microbleeds: systematic review, subgroup analyses and standards for study design and reporting. Brain 2007;130:1988-2003.

4. Kato H, Izumiyama M, Izumiyama $\mathrm{K}$, et al. Silent cerebral microbleeds on T2* weighted MRl: correlation with stroke subtype, stroke recurrence, and leukoaraiosis. Stroke 2002;33:1536-40.

5. Gao T, Wang Y, Zhang Z. Silent cerebral microbleeds on susceptibility-weighted imaging of patients with ischemic stroke and leukoaraiosis. Neurol Res 2008;30:272-6.

6. Naka H, Nomura E, Takahashi T, et al. Combinations of the presence or absence of cerebral microbleeds and advanced white matter hyperintensity as predictors of subsequent stroke types. Am J Neuroradiol 2006;27:830-5.

7. Won SS, Hwa LB, Kim EJ, et al. Clinical significance of microbleeds in subcortical vascular dementia. Stroke 2007;38:1949-51.

8. Werring DJ, Frazer DW, Coward LJ, et al. Cognitive dysfunction in patients with cerebral microbleeds on T2*-weighted gradient-echo MRI. Brain 2004;127:2265-75.

9. Kim JS, Choi KS. Poststroke depression and emotional incontinence: correlation with lesion location. Neurology 2000;54:1805-10.

10. Andersen G, Vestergaard K, Riis JO. Citalopram for post-stroke pathological crying Lancet 1993:342:837-9.

11. Schiffer R, Pope LE. Review of pseudobulbar affect including a novel and potential therapy. J Neuropsychiatry Clin Neurosci 2005;17:447-54.

12. Dark FL, McGrath JJ, Ron MA. Pathological laughing and crying. Aust N Z J Psychiatry 1996;30:472-9

13. Robinson RG, Parikh RM, Lipsey JR, et al. Pathological laughing and crying following stroke: validation of a measurement scale and a double-blind treatment study. Am J Psychiatry 1993;150:286-93

14. Tang WK, Chan SSM, Chiu HFK, et al. Emotional incontinence in Chinese stroke patients: diagnosis, frequency, and clinical and radiological correlates. J Neurol 2004:251:865-9

15. Kim JS. Pathologic laughter after unilateral stroke. J Neurol Sci 1997;148:121-5.

16. Ceccaldi M, Poncet M, Milandre L, et al. Temporary forced laughter after unilateral strokes. Eur Neurol 1994;34:36-9.

17. Brown KW, Sloan RL, Pentland B. Fluoxetine as a treatment for post-stroke emotionalism. Acta Psychiatr Scand 1998;98:455-8.

18. Wilson SAK. Some problems in neurology. J Neurol Psychopathol 1924:4:299-333.

19. Parvizi J, Anderson SW, Martin CO, et al. Pathological laughter and crying: a link to the cerebellum. Brain 2001;124:1708-19.

20. Black DW. Pathological laughter: a review of literature. J Nerv Ment Dis 1982;170:67-71.

21. House A, Dennis M, Molyneux A, et al. Emotionalism after stroke. BMJ 1989:298:991-4
22. Langworthy $\mathbf{O R}$, Hesser FH. Syndrome of pseudobulbar palsy. Anatomic and physiologic analysis. Arch Intern Med 1940;64:106-21.

23. Malloy $\mathbf{P}$, Bihrle A, Duffy J. The orbitomedial frontal syndrome. Arch Clin Neuropsychol 1993:8:185-201.

24. Ghaffar 0, Chamelian L, Feinstein A. Neuroanatomy of pseudobulbar affect: a quantitative MRI study in multiple sclerosis. J Neurol 2008;255:406-12.

25. Morris PLP, Robinson RG, Raphael B. Emotional lability after stroke. Aust N Z J Psychiatry 1993;27:601-5.

26. Ross ED, Stewart RS. Pathological display of affect in patients with depression and right frontal brain damage. An alternative mechanism. J Nerv Ment Dis 1987; 175:165-72.

27. Kim JS. Post-stroke emotional incontinence after small lenticulocapsular stroke: Correlation with lesion location. J Neurol 2002;49:805-10.

28. Asfora WT, DeSalles AA, Abe M, et al. Is the syndrome of pathological laughing and crying a manifestation of pseudobulbar palsy? J Neurol Neurosurg Psychiatry 1989;52:523-5.

29. Cummings JL. Frontal-subcortical circuits and human behaviors. Arch Neurol 1993:50:873-80.

30. Tekin S, Cummings JL. Frontal-subcortical neuronal circuits and clinica neuropsychiatry: an update. J Psychosom Res 2002;53:647-54.

31. Carrera E, Bogousslavsky J. The thalamus and behavior: effects of anatomically distinct strokes. Neurology 2006;66:1817-23.

32. Brott T, Marler JR, Olinger CP, et al. Measurement of acute cerebral infarctions: a clinical examination scale. Stroke 1989;20:864-70.

33. Choi-Kwon S, Han SW, Kwon SU, et al. Fluoxetine treatment in poststroke depression, emotional incontinence, and anger proneness: a double-blind, placebocontrolled study. Stroke 2006;37:156-61.

34. Chiu HFK, Lee HCB, Chung D. Reliability and validity of the Cantonese version of the Mini-Mental State Examination: a preliminary study. Hong Kong J Psychiatry 1994:4:25-8.

35. Mok VC, Wong A, Lam WW, et al. Cognitive impairment and functional outcome after stroke associated with small vessel Dis. J Neurol Neurosurg Psychiatry 2004:5:560-6.

36. Dichgans $\mathbf{M}$, Holtmannspotter $\mathbf{M}$, Herzog J, et al. Cerebral microbleeds in CADASIL: a gradient-echo magnetic resonance imaging and autopsy study. Stroke 2002;33:67-71

37. Carrera E, Michel P, Bogousslavsky J. Anteromedian, central and posterolatera territory infarcts of the thalamus. Three variant types. Stroke 2004;35:2826-34.

38. Fazekas F, Chawluk JB, Alavi A, et al. MR signal abnormalities at $1.5 \mathrm{~T}$ in Alzheimer's dementia and normal aging. Am J Roentgenol 1987:149:351-6.

39. Tanaka A, Ueno Y, Nakayama Y, et al. Small chronic hemorrhages and ischemic lesions in association with spontaneous intracerebral hematomas. Stroke 1999;30:1637-42.

40. Guigere M, Goldman-Rakic PS. Mediodorsal nucleus: areal, laminar, and tangential distribution of afferents and efferents in the frontal lobe of rhesus monkey. $J$ Comp Neurol 1988;277:195-213

41. Groenewegen HJ, Berendse HW. The specificity of the "nonspecific" midline and intralaminar nuclei. Trends Neurosci 1994:17:52-6.

42. Bogousslavsky J, Ferrazzini M, Regli F, et al. Manic delirium and frontal-like syndrome with paramedian infarction of the right thalamus. J Neurol Neurosurg Psychiatry 1988;51:116-19.

43. Gilchrist I, Goldstein LH, Jadresic D, et al. Thalamo-frontal psychosis. Br J Psychiatry 1993:163:113-15.

44. Schmahmann JD. Vascular syndromes of the thalamus. Stroke 2003;34:2264-78

45. McCullagh S, Moore M, Gawel M, et al. Pathological laughing and crying in amyotrophic lateral sclerosis: an association with prefrontal cognitive dysfunction. J Neurol Sci 1999;169:43-8.

46. Tang WK, Sandra S, Chan M, et al. Frequency and clinical determinants of poststroke dementia in Chinese. Stroke 2004;18:98-103.

47. Verdelho A, Henon H, Lebert F, et al. Depressive symptoms after stroke and relationship with dementia: a three-year follow-up study. Neurology 2004:62:905-11. 